\title{
Baseline serum TSH and risk of thyroid microcarcinoma \\ in non-toxic nodular thyroid disease
}

\section{Dimitrios Askitis ${ }^{1}$, Eleni I. Efremidou 1, Michael Karanikas ${ }^{1}$, Grigorios Tripsianis ${ }^{1}$, Alexandros Polychronidis ${ }^{1}$, Nikolaos}

\author{
Lyratzopoulos 1
}

${ }^{1}$ First Surgical Department, University Hospital of Alexandroupolis, Greece

\section{Introduction- Objective}

Thyroid cancer comprises the most common endocrine malignancy and a variety of studies have examined the role of $\mathrm{TSH}$ as an independent risk factor for the manifestation of differentiated thyroid cancer in otherwise benign thyroid disorders. ${ }^{1,2}$ Objective of the current retrospective study was the assessment of a possible relation between baseline serum TSH and incidental thyroid microcarcinoma (mTC $<10 \mathrm{~mm}$ ) diagnosed after total thyroidectomy (TT), in a patient cohort with non-toxic thyroid disorders, without any preoperative suspicion or cytological establishment of thyroid malignancy.

\section{Patients and methods}

A 5-year retrospective study (2005-2010) was conducted in a total of 186 patients (146 female/ 40 male) who underwent TT indicated for non-toxic nodular thyroid diseases (non toxic multinodular goiter- NTMG or non functioning solitary thyroid nodule- STN). The pre-op diagnosis was NTMG in $152 / 186$ pts $(81.7 \%)$ and STN in $34 / 186$ pts $(18.3 \%)$. All surgical specimens were histopathologically examined at the University Pathology Department. Pre-op TSH levels were estimated in all patients as median value and interquartile range (laboratory's reference range 0.38-3.8 $\mu \mathrm{IU} / \mathrm{ml})$ and results were evaluated regarding pre-op diagnosis and also the finding of incidental $\mathrm{mTC}$. $\mathrm{P}$ value $<0.05$ was concerned as statistically significant.

\section{Results}

Incidental mTC was diagnosed in $32 / 186$ pts $(17.2 \%)$ (rate females:males 2.2:1)- group A. In the rest 154/186 pts $(82.8 \%)$ - group B a benign thyroid disorder was established. The median value of baseline serum TSH was higher in group A but not statistically significant compared to group B (1.02 vs $0.80 ; p=0.293)$. Regarding pre-op diagnosis, STN pts were found with elevated TSH in group $B(1.3$ vs $0.83 ; p=0.289)$, whereas pts with NTMG had higher TSH levels in group $A$. This last finding was marginally non statistically significant between group $A$ and group $B(1.16$ vs $0.75 ; p=0.05)$.

\section{DIAGNOSIS}

$\begin{array}{lr}\text { STN } & 34 \\ \text { NTMG } & 152\end{array}$

Total

186

The comparison of median TSH values between the groups of histopathologically established benignity and malignancy, totally and regarding preoperative diagnosis, gave the following results:

$\begin{array}{llcl} & \text { BENIGNITY } & \text { MALIGNANCY } & \boldsymbol{p} \text { value } \\ \text { TOTAL TSH }(\boldsymbol{\mu} \mathrm{IU} / \mathrm{mI}) & 0.8(0.5-1.42) & 1.02(0.56-1.84) & 0.293 \\ \text { STN } & 1.3(0.73-2.15) & 0.83(0.6-1.73) & 0.289 \\ \text { NTMG } & 0.75(0.48-1.23) & 1.16(0.65-1.7) & \mathbf{0 . 0 5}\end{array}$

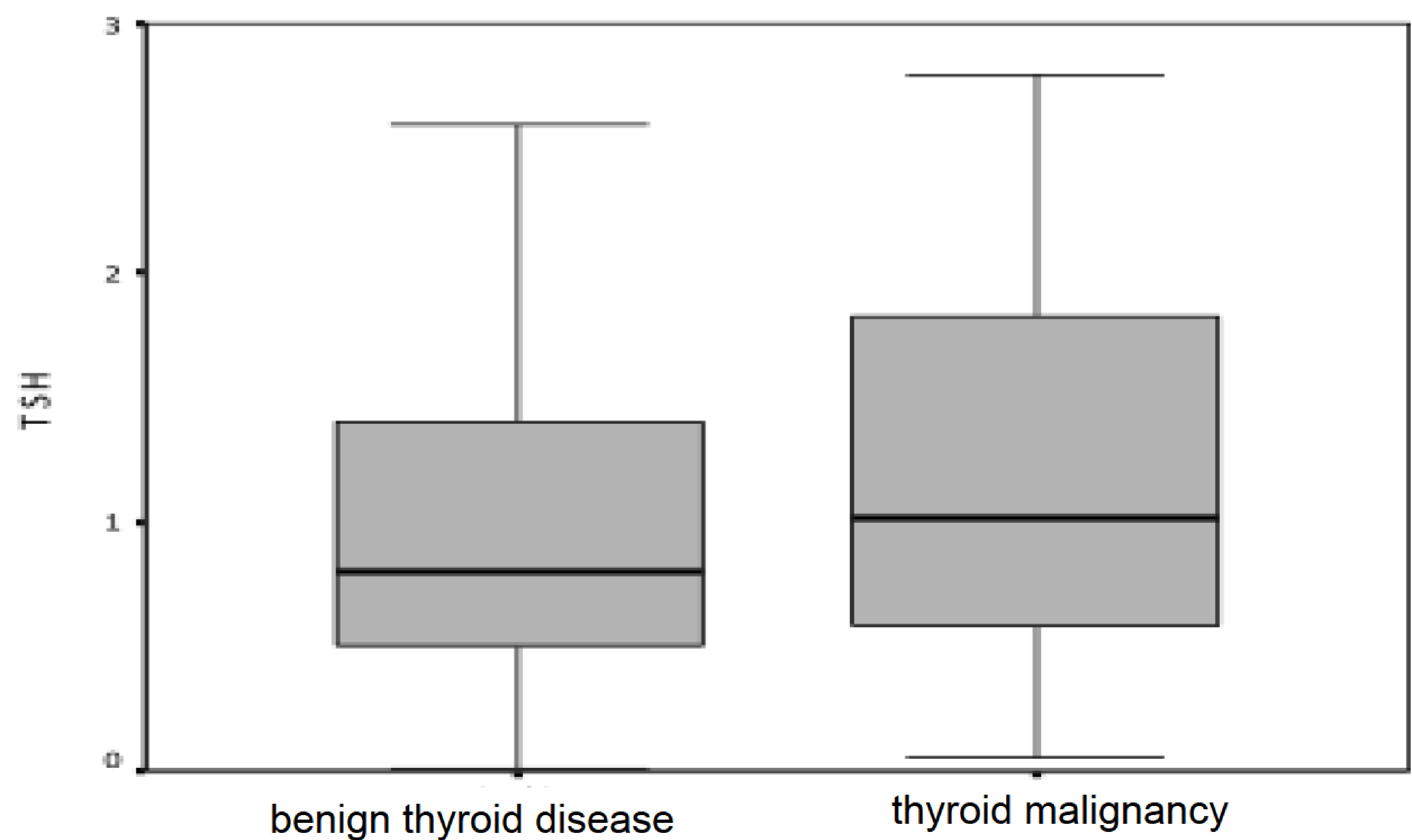

\section{Conclusions}

Elevated basal serum TSH has not been proven as an independent risk predictor for the co-existence of thyroid microcarcinoma in non-toxic nodular thyroid diseases. However, a significant trend of higher TSH levels was shown in non-toxic multinodular goiter harbouring malignancy.

Further clinical studies evaluating the role of TSH in thyroid cancer may be required.

Literature: 1. Boelaert K. The association between serum TSH concentration and thyroid cancer. Endocr Relat Cancer. 2009 Dec;16(4): 1065-72

2. Shi L1, Li Y, Guan H, Li C, Shi L, Shan Z, Teng W. Usefulness of serum thyrotropin for risk prediction of differentiated thyroid cancers does not apply to microcarcinomas: results of 1,870 Chinese patients with thyroid nodules. Endocr J. 2012;59(11): 973-80 